\title{
The Klein-Gordon Equation with Light-Cone Data
}

\section{Lars-Erik Lundberg}

Matematisk Institut HC $\varnothing$, DK-2100 Copenhagen $\varnothing$, Denmark

\begin{abstract}
It is shown that the characteristic Cauchy problem $\left(\frac{\partial^{2}}{\partial t^{2}}-\Delta+1\right)$ $\cdot u(x, t)=0, u(x,-|x|)=f(x), x \in \mathbb{R}^{n}, n \geqq 1$ has a unique finite energy weak solution for all $f$ such that $\int d x\left(|\nabla f|^{2}+|f|^{2}\right)<\infty$ and all finite energy weak solutions of the equation are obtained in this way.
\end{abstract}

\section{Introduction}

We shall consider the characteristic Cauchy problem for the Klein-Gordon $(K-G)$ equation

$$
\left\{\begin{array}{l}
\left(\frac{\partial^{2}}{\partial t^{2}}-\Delta+1\right) u(x, t)=0 \\
u(x,-|x|)=f(x)
\end{array}\right.
$$

where $x \in \mathbb{R}^{n}, n \geqq 1$ and $t \in \mathbb{R}$. We shall prove that this problem has a unique finite energy weak solution for all $f$ such that $\int_{\mathbb{R}^{n}} d x\left(|\nabla f|^{2}+|f|^{2}\right)<\infty$, and all finite energy weak solutions of (1.1) are obtained in this way. In fact the energy $E$ fulfills

$$
\begin{aligned}
E & \equiv 1 / 2 \int_{\mathbb{R}^{n}} d x\left(\left|\frac{\partial u}{\partial t}(x, t)\right|^{2}+|\nabla u(x, t)|^{2}+|u(x, t)|^{2}\right) \\
& =1 / 2 \int_{\mathbb{R}^{n}} d x\left(|\nabla f|^{2}+|f|^{2}\right) .
\end{aligned}
$$

We shall also give an explicit formula for $u(x, t)$ in terms of its light-cone restriction $f$ with the help of a "light-cone Fourier transform".

For some general results on characteristic Cauchy-problems see Hörmander [1]. The wave-equation has been considered by Riesz [2] and Strichartz [3]. 
They consider the wave-equation inside the light-cone and prove that the problem

$$
\left\{\begin{array}{l}
\left(\frac{\partial^{2}}{\partial t^{2}}-\Delta\right) u(x, t)=0,|x|<-t, t<0 \\
u(x,-|x|)=f(x)
\end{array}\right.
$$

has a unique weak solution for all $f$ such that

$$
\int_{\mathbb{R}^{n}} \frac{d x}{|x|}|f|^{2}<\infty
$$

In the $K-G$ case we get global uniqueness and our approach is very different from theirs.

In Section 2 we recall some simple properties of smooth solutions to the $K-G$ equation in particular two local conservation equations are given. The behaviour of a smooth solution along a light-cone is considered in Section 3. In Section 4 this together with one of the conservation equations is used to express the energyintegral as an integral of the solution over the light-cone [Eq. (1.3)].

The $K-G$ equation is written in terms of light-cone coordinates in Section 5 for $n \geqq 3$ and in Section 6 the spectral theory is developed including a so-called "light-cone Fourier transform". In Section 7 the general case $n \geqq 1$ is considered by expressing the $K-G$ equation in terms of sesquilinear forms. We end with conclusions in Section 8.

\section{Local Conservation Equations}

In this section we review some well-known properties of smooth solutions (defined below) to the Klein-Gordon $(K-G)$ equation in $n+1$ space-time dimensions,

$$
(\square+1) u=\left(\frac{\partial^{2}}{\partial t^{2}}-\Delta+1\right) u=0
$$

With smooth solutions we shall mean those solutions whose initial data $\left\{u(x, 0), \frac{\partial u}{\partial t}(x, 0)\right\}$ are both in the Schwartz-space $\mathscr{S}\left(\mathbb{R}^{n}\right)$. This means that a smooth solution can always be written

$$
u(x, t)=(2 \pi)^{-n / 2} \int_{\mathbb{R}^{n}} \frac{d k}{\omega_{k}}\left[e^{-i \omega_{k} t+i k \cdot x} \hat{f}_{+}(k)+e^{i \omega_{k} t+i k \cdot x} \hat{f}_{-}(k)\right],
$$

where $\omega_{k}=\sqrt{|k|^{2}+1}$ and $\hat{f}_{ \pm}(k) \in \mathscr{S}\left(\mathbb{R}^{n}\right)$.

Notation. Let $x^{\mu}, \mu=0,1, \ldots, n$ be the components of the $n+1$ dimensional vector $(t, x), t \in \mathbb{R}, x \in \mathbb{R}^{n}$ and let $x_{\mu}$ denote the components of $(t,-x)$. Let furthermore $\nabla^{\mu}$ $=\frac{\partial}{\partial x_{\mu}}$ and $\nabla_{\mu}=\frac{\partial}{\partial x^{\mu}}$. We note that $\square=\nabla^{\mu} \nabla_{\mu}$ (summation convention) and that $\nabla_{\mu}$ are the components of the $n+1$ dimensional gradient $\left(\frac{\partial}{\partial t}, \nabla\right)$. 
Definition 2.1. Let $u$ and $v$ be two smooth solutions of $(\square+1) u=0$ and let $J_{\mu}$, and $T_{\mu}$ be given by

$$
\begin{aligned}
J_{\mu} & =i\left(\bar{v} \nabla_{\mu} u-\left(\nabla_{\mu} \bar{v}\right) u\right), \\
T_{\mu} & =\left(\bar{v}_{t} u_{t}+(\nabla \bar{v}) \cdot(\nabla u)+\bar{v} u, \bar{v}_{t} \nabla u+(\sqrt{v}) u_{t}\right)_{\mu},
\end{aligned}
$$

where $u_{t}=\frac{\partial u}{\partial t}$.

It is a simple consequence of the equation $(\square+1) u=0$ that

$$
\nabla^{\mu} J_{\mu}=0, \quad \nabla^{\mu} T_{\mu}=0
$$

which are usually called local conservation equations.

If we choose $v=u$ and then integrate the equation $\nabla^{\mu} T_{\mu}=0$ over the region in $\mathbb{R}^{n+1}$ between two different hypersurfaces $t=$ constant, it follows from Gauss's theorem that the so-called energy integral

$$
E=\frac{1}{2} \int_{\mathbb{R}^{n}} d x\left(\left|u_{t}(x, t)\right|^{2}+|\nabla u(x, t)|^{2}+|u(x, t)|^{2}\right),
$$

is independent of $t$. By integrating $\nabla^{\mu} J_{\mu}=0$ similarily we find that

$$
\sigma(v, u)=\frac{i}{2} \int_{\mathbb{R}^{n}} d x\left(\bar{v}(x, t) u_{t}(x, t)-\bar{v}_{t}(x, t) u(x, t)\right),
$$

is also independent of $t$.

If (2.1) is inserted into (2.5) a simple calculation gives

$$
E=\int_{\mathbb{R}^{n}} d k\left(\left|\hat{f}_{+}\right|^{2}+\left|\hat{f}_{-}\right|^{2}\right)
$$

and similarily we get for $\sigma(u, u)$

$$
\sigma(u, u)=\int_{\mathbb{R}^{n}} \frac{d k}{\omega_{k}}\left(\left|\hat{f}_{+}\right|^{2}-\left|\hat{f}_{-}\right|^{2}\right)
$$

\section{Decay of Solution Along Light-Cones}

Let $u$ be a solution of $(\square+1) u=0$ with data $\left\{u, u_{t}\right\} \in C_{0}^{\infty} \times C_{0}^{\infty}$ (infinitely differentiable and compact support) at $t=0$. Let furthermore $p^{\mu}$ be the components of a vector $\left(p^{0}, p\right) \in \mathbb{R}^{n+1}$ with $\left(p^{0}\right)^{2}-|p|^{2}<0$, i.e. it is a space-like vector. It then follows from the finite propagation velocity that $u\left(\lambda p, \lambda p^{0}\right), \lambda \in \mathbb{R}$ has compact support in $\lambda$, that is $u(x, t)$ has compact support in space-like directions. One can now ask how $u(x, t)$ behaves in light-like directions, i.e. when $\left(p^{0}\right)^{2}-|p|^{2}=0$.

The solution $u(x, t)$ has the representation (2.1) with $\hat{f}_{ \pm} \in \mathscr{S}\left(\mathbb{R}^{n}\right)$, i.e. it is sufficient to consider the behaviour of

$$
v(x)=\int d k e^{i \omega_{k}|x|+i k \cdot x} g(k),
$$

with $g \in \mathscr{S}$, for $|x|$ large. 
Proposition 3.1. For each $m \in \mathbb{N}$ there exists constants $c_{m}$ and $d_{m}$ such that

$$
\left(1+|x|^{m}\right)|v(x)| \leqq c_{m}, \quad\left(1+|x|^{m}\right)|\nabla v(x)| \leqq d_{m} .
$$

Proof. Choose the coordinate system such that $x_{2}=\ldots=x_{n}=0$ and $x_{1} \geqq 0$, i.e.

$$
v(x)=\int d k e^{i\left(\omega_{k}+k_{1}\right) x_{1}} g(k),
$$

Let us then introduce $\xi=\omega_{k}+k_{1}$ as integration variable instead of $k_{1}$ that is

$$
k_{1}=\frac{1}{2 \xi}\left(-\sum_{i=2}^{n} k_{i}^{2}-1+\xi^{2}\right), \quad d k_{1}=d \xi \frac{1}{2 \xi^{2}}\left(\sum_{i=2}^{n} k_{i}^{2}+1+\xi^{2}\right),
$$

and we get

$$
v(x)=\int_{-\infty}^{\infty} d k_{2} \ldots d k_{n} \int_{0}^{\infty} d \xi e^{i \xi x_{1}} h\left(\xi, k_{2}, \ldots, k_{n}\right),
$$

with

$$
h\left(\xi, k_{2}, \ldots, k_{n}\right)=\frac{1}{2 \xi^{2}}\left(\sum_{i=2}^{n} k_{i}^{2}+1+\xi^{2}\right) g\left(\frac{1}{2 \xi}\left(-\sum_{i=2}^{n} k_{i}^{2}-1+\xi^{2}\right), k_{2}, \ldots, k_{n}\right) .
$$

It follows from this formula that $h$ extends to all $\xi \in \mathbb{R}$ in a natural way and that $h \in \mathscr{S}\left(\mathbb{R}^{n}\right)$. It also follows from (3.5) that $\frac{\partial^{p} h}{\partial \xi^{p}}=0$ at $\xi=0$ for all $p=0,1,2, \ldots$, due to the fact that $g \in \mathscr{S}\left(\mathbb{R}^{n}\right)$. Partial integration with respect to $\xi$ in (3.4) gives the first part of the proposition (the uniformity of the fall off in all directions follows easily).

For the proof of the second part we note that

$$
\nabla v(x)=i \int d k\left(\omega_{k} e+k\right) e^{i \omega_{k}|x|+i k \cdot x} g(k),
$$

where $e=\frac{x}{|x|}$ and the second estimate follows in complete analogy with the one above.

\section{The Light-Cone (LC) Energy Integral and the Hilbert-Space $\mathscr{H}$ of LC Data}

In this section we shall express the energy $E$ given by (2.5) as an integral over a light-cone, in fact we shall prove the following.

\section{Proposition 4.1.}

Let $u$ be a smooth solution of the $K-G$ equation $(\square+1) u=0$. It then follows that

$$
\begin{aligned}
E & =\frac{1}{2} \int d x\left(\left|u_{t}(x, 0)\right|^{2}+|\nabla u(x, 0)|^{2}+|u(x, 0)|^{2}\right) \\
& =\frac{1}{2} \int d x\left(|\nabla f(x)|^{2}+|f(x)|^{2}\right),
\end{aligned}
$$

where $f(x)=u(x,-|x|)$.

Proof. Let $\Omega=\left\{(x, t) ; t \leqq 0, t^{2}-|x|^{2} \leqq 0\right\}$. Let us then consider a solution $u(x, t)$ with compact support at $t=0$ and put $v=u$ in the expression for $T_{\mu}$ given by (2.3). We then integrate the equation $\nabla^{\mu} T_{\mu}=0$ over $\Omega$. Let $C=\{(x, t) ; t=-|x|\}$, and let 
$\left(e^{0}, e\right)$ be the upward unit normal to $C$. Gauss's theorem then gives

$$
\begin{aligned}
E & =\frac{1}{2} \int_{t=0} d x\left(\left|u_{t}\right|^{2}+|\nabla u|^{2}+|u|^{2}\right) \\
& =\frac{1}{2} \int_{C} d C\left\{\left(\left|u_{t}\right|^{2}+|\nabla u|^{2}+|u|^{2}\right) e^{0}-\left(\bar{u}_{t} \nabla u+(\nabla \bar{u}) u_{t}\right) \cdot e\right\},
\end{aligned}
$$

where $d C=\sqrt{2} d x$. It is convenient to absorb the $\sqrt{2}$ in $\left(e^{0}, e\right)$, i.e. $\left(e^{0}\right)^{2}+|e|^{2}=2$, $e^{0}=1$ and $e=\frac{x}{|x|}$. By completing the square on the r.h.s. of (4.2) we get

$$
E=\frac{1}{2} \int_{C} d x\left\{\left|\frac{\partial u}{\partial e}\right|^{2}+|D u|^{2}+|u|^{2}\right\}
$$

where $\frac{\partial}{\partial e}=e^{\mu} \nabla_{\mu}$ (the co-normal derivative) and $D=\nabla-e(e \cdot \nabla)$. Let $f(x)=u(x,-|x|)$. It then follows that $\frac{\partial u}{\partial e}(x, t) \mid=-e \cdot \nabla f(x)$, and $D u(x, t) \mid=D f(x)$. Inserting this into (4.3) finally gives (4.1).

The vanishing of the surface term at infinity and the convergence of the r.h.s. of (4.1) follows from the results in the previous section.

Equation (4.1) implies that the solution $u(x, t)$ is uniquely determined by its restriction $f$ to $C$.

Definition 4.2. Let $\mathscr{H}$ denote the completion of $C_{0}^{\infty}\left(\mathbb{R}^{n}\right)$ in the norm

$$
\|f\|_{\mathscr{H}}^{2}=\frac{1}{2} \int_{\mathbb{R}^{n}} d x\left(|\nabla f|^{2}+|f|^{2}\right)
$$

i.e. $\mathscr{H}$ is a Hilbert space [the so called Sobolev space $\left.H^{1}\left(\mathbb{R}^{n}\right)\right]$.

Let $u(x, t)$ be a smooth solution of the $K-G$ equation. It then follows from (2.1) that $f(x)=u(x,-|x|)$ is given by

$$
f(x)=(2 \pi)^{-n / 2} \int_{\mathbb{R}^{n}} \frac{d k}{\omega_{k}}\left[e^{i \omega_{k}|x|+i k \cdot x} \hat{f}_{+}(k)+e^{-i \omega_{k}|x|+i k \cdot x} \hat{f}_{-}(k)\right],
$$

Equations (2.7) and (4.1) give

$$
\|f\|_{\mathscr{H}}^{2}=\int_{\mathbb{R}^{n}} d k\left(\left|\hat{f}_{+}\right|^{2}+\left|\hat{f}_{-}\right|^{2}\right), \hat{f}_{ \pm} \in \mathscr{S}\left(\mathbb{R}^{n}\right)
$$

which is a kind of Plancherel's theorem which means that the mapping $\left\{\hat{f}_{+}, \hat{f}_{-}\right\} \rightarrow f$ extends to an isometry from $L^{2}\left(\mathbb{R}^{n}\right) \oplus L^{2}\left(\mathbb{R}^{n}\right)$ into $\mathscr{H}$.

In the following sections we shall prove that this isometry maps onto $\mathscr{H}$.

By integrating the equation $\nabla^{\mu} J_{\mu}=0$ over $\Omega$ (in analogy with the proof of Proposition 4.1) we get

$$
\begin{aligned}
\sigma(v, u) & =\frac{i}{2} \int_{\mathbb{R}^{n}} d x\left(\bar{v}(x, t) u_{t}(x, t)-\bar{v}_{t}(x, t) u(x, t)\right) \\
& =\frac{i}{2} \int_{C} \frac{d x}{|x|}((x \cdot \nabla \bar{g}) f-\bar{g} x \cdot \nabla f),
\end{aligned}
$$

where $f(x)=u(x,-|x|)$ and $g(x)=v(x,-|x|)$. 


\section{The K-G Equation in LC Coordinates for $n \geqq 3$}

In this section we shall express the K-G equation in "light-cone coordinates" for $n \geqq 3$.

Definition 5.1. Let us consider the coordinate transformation $\{x, t\} \rightarrow\{x, \tau\}$ where $\tau=t+|x|$. Thus a $\tau=$ constant surface is the backward light-cone with apex at $(0, \tau)$. Let us furthermore put $\tilde{u}(x, \tau)=u(x,-|x|+\tau)$.

It then follows that $\nabla \tilde{u}=\nabla u-u_{t} e$, where $e=\frac{x}{|x|}$ and $\frac{\partial \tilde{u}}{\partial \tau}=u_{t}$. The K-G equation can then be written

$$
\left(\frac{\partial^{2}}{\partial t^{2}}-\Delta+1\right) u=\left(\frac{\partial^{2}}{\partial \tau^{2}}-\left(\nabla+e \frac{\partial}{\partial \tau}\right)^{2}+1\right) \tilde{u}=0
$$

i.e. the second order $\tau$-derivative cancels and we get

$$
-i(\nabla \cdot e+e \cdot \nabla) i \frac{\partial \tilde{u}}{\partial \tau}=(-\Delta+1) \tilde{u} .
$$

Definition 5.2. Put $S_{0}=-i(\nabla \cdot e+e \cdot \nabla)$ with $\mathscr{D}\left(S_{0}\right)=C_{0}^{\infty}\left(\mathbb{R}^{n}\right)$ and $T_{0}=-\Delta+1$ with $\mathscr{D}\left(T_{0}\right)=C_{0}^{\infty}\left(\mathbb{R}^{n}\right)$ considered as operators in $L^{2}\left(\mathbb{R}^{n}\right) . T_{0}$ is essentially self-adjoint. Let $T$ denote the closure of $T_{0}$ in $L^{2}$.

We note that the Hilbert-space $\mathscr{H}$ given in Definition 4.2 can be identified with $\mathscr{D}\left(T^{1 / 2}\right)$ in $L^{2}\left(\mathbb{R}^{n}\right)$ with

$$
(f, g)_{\mathscr{H}}=\frac{1}{2}\left(T^{1 / 2} f, T^{1 / 2} g\right)_{2},
$$

for $f, g \in \mathscr{H}=\mathscr{D}\left(T^{1 / 2}\right)$.

It is easily verified that $\left(S_{0} f\right)(x)=-2 i \frac{1}{r^{\alpha}} \frac{\partial}{\partial r}\left(r^{\alpha} f(x)\right)$, where $r=|x|, \alpha=\frac{n-1}{2}$ and $f \in \mathscr{D}\left(S_{0}\right)$.

Proposition 5.3. For $n \geqq 3$ the following inequality holds

$$
\left\|S_{0} f\right\|_{2}^{2} \leqq 4 \cdot \int_{\mathbb{R}^{n}}|\nabla f|^{2} d x, f \in \mathscr{D}\left(S_{0}\right) .
$$

Proof. We get

$$
\begin{aligned}
\left\|S_{0} f\right\|_{2}^{2} & =4 \int d r d \omega\left|\frac{\partial}{\partial r}\left(r^{\alpha} f\right)\right|^{2}=4 \int d r d \omega r^{\alpha} \bar{f}\left(-\frac{\partial^{2}}{\partial r^{2}}\left(r^{\alpha} f\right)\right) \\
& =4 \int r^{n-1} d r d \omega \bar{f}\left(-\frac{\partial^{2} f}{\partial r^{2}}-\frac{n-1}{r} \frac{\partial f}{\partial r}-\frac{\alpha(\alpha-1)}{r^{2}} f\right) \\
& \leqq 4 \int r^{n-1} d r d \omega \bar{f}\left(-\frac{\partial^{2} f}{\partial r^{2}}-\frac{n-1}{r} \frac{\partial f}{\partial r}\right) \leqq 4 \int d x|\nabla f|^{2} .
\end{aligned}
$$

Due to the inequality (5.4) we can extend $S_{0}$ to $\mathscr{D}\left(T^{1 / 2}\right)$, and let this extension be denoted $S$, which also is symmetric in $L^{2}$.

The K-G equation can then be written

$$
i S \frac{d \tilde{u}}{d \tau}=T \tilde{u},
$$

and it is now tempting to try to invert $S$, which in fact we shall do. 
Proposition 5.4. The range of $S_{0}$ is dense in $L^{2}$.

Proof. We will prove the proposition by showing that the nullspace of $S_{0}^{*}$ in $L^{2}$ is trivial. It follows that $\mathscr{D}\left(S_{0}^{*}\right)=\left\{f \in L^{2} ; \frac{1}{r^{\alpha}} \frac{\partial}{\partial r}\left(r^{\alpha} f\right) \in L^{2}\right\}$. Let us then consider all solutions of $\frac{1}{r^{\alpha}} \frac{\partial}{\partial r}\left(r^{\alpha} f\right)=0$ which lie in $L_{\text {loc }}^{2}$, i.e. $f(x)=\frac{c(e)}{r^{\alpha}}$ where $c$ is square integrable on the unit sphere. These solutions are not globally in $L^{2}$.

This means that $S$ has a densely defined inverse in $L^{2}$, i.e.

$$
\left(S^{-1} g\right)(x)=\frac{i}{2 r^{\alpha}} \int_{0}^{r} \varrho^{\alpha} g(\varrho, e) d \varrho,
$$

with $g \in \mathscr{D}\left(S^{-1}\right)=\mathscr{R}(S)$.

Proposition 5.5. $T^{-1} S$ defines a continuous self-adjoint operator on $\mathscr{H}$ with a dense range.

Proof. Proposition 5.3 implies that $\|S f\|_{2}^{2} \leqq 8\|f\|_{\mathscr{H}}^{2}$ for all $f \in \mathscr{H}$. This gives

$$
\left\|T^{-1} S f\right\|_{\mathscr{H}}^{2}=\frac{1}{2}\left\|T^{-1 / 2} S f\right\|_{2}^{2} \leqq \frac{1}{2}\left\|T^{-1 / 2}\right\|_{2}^{2}\|S f\|_{2}^{2} \leqq 4\|f\|_{\mathscr{H}}^{2},
$$

and the symmetry follows from

$$
\left(f, T^{-1} S g\right)_{\mathscr{H}}=\frac{1}{2}(f, S g)_{2}=\frac{1}{2}(S f, g)_{2}=\left(T^{-1} S f, g\right)_{\mathscr{H}},
$$

for all $f, g \in \mathscr{H}$. Let us then assume that there is a vector $\psi \in \mathscr{H}$ such that $\left(\psi, T^{-1} S f\right)_{\mathscr{H}}=0$ for all $f \in \mathscr{H}$. This implies that $(\psi, S f)_{2}=0$, i.e. $\psi=0$ due to Proposition 5.4.

Definition 5.6. Let $\mathscr{D}(A)=T^{-1} S \mathscr{H}$ and $A: \mathscr{D}(A) \rightarrow \mathscr{H}$ be given by $A=S^{-1} T$.

The following theorem is a direct consequence of Proposition 5.4.

Theorem 5.7. $A: \mathscr{D}(A) \rightarrow \mathscr{H}$ is self-adjoint.

This means that the K-G Equation (5.5) can be written

$$
i \frac{d \tilde{u}}{d \tau}=A \tilde{u},
$$

and we now specify that $\tilde{u} \in \mathscr{D}(A)$, i.e. a solution is given by $\tilde{u}(\tau)=e^{-i A \tau} f, f \in \mathscr{D}(A)$.

\section{Spectral Theory for $\boldsymbol{A}$ and the LC Fourier Transform}

In this section we shall rederive the Plancherel's type formula (4.6)

$$
\|f\|_{\mathscr{H}}^{2}=\left\|\hat{f}_{+}\right\|_{2}^{2}+\left\|\hat{f}_{-}\right\|_{2}^{2},
$$

with the difference that this time the $f$ 's on the 1.h.s. belongs to a dense subset in $\mathscr{H}$. 
Let $\{E(\lambda)\}$ be the spectral family associated with $A$ i.e. $A=\int_{-\infty}^{\infty} \lambda d E(\lambda)$. The following well-known formula allows us to express $E(\lambda)$ in terms of the resolvent $R(z)=(A-z)^{-1}$.

$$
\begin{aligned}
& \left(f,\left[\frac{1}{2}(E(\beta)+E(\beta-0))-\frac{1}{2}(E(\alpha)+E(\alpha-0))\right] f\right)_{\mathscr{H}} \\
& =\lim _{\varepsilon \downarrow 0} \frac{1}{2 \pi i} \int_{\alpha}^{\beta} d \mu(f,[R(\mu+i \varepsilon)-R(\mu-i \varepsilon)] f)_{\mathscr{H}} .
\end{aligned}
$$

We shall evaluate the r.h.s. of (6.2). For that we need a more explicit formula for $R(z)$.

Consider the equation

$$
(A-z) g=f, \quad f \in \mathscr{H}, \quad g \in \mathscr{D}(A),
$$

for $\operatorname{Im} z \neq 0$. Let us consider this equation as an equation in $L^{2}$ and apply $S$ to it

$$
(T-z S) g=S f \in L^{2} .
$$

Definition 6.1. Let $V_{z}: C_{0}^{\infty} \rightarrow L^{2}$ be given by

$$
\left(V_{z} \varphi\right)(x)=e^{-i z|x|} \varphi(x) .
$$

This operator extends to a bounded operator for $\operatorname{Im} z<0$, to a unitary operator for $\operatorname{Im} z=0$ and to an unbounded operator for $\operatorname{Im} z>0$.

Proposition 6.2. For $\varphi \in C_{0}^{\infty}\left(\mathbb{R}^{n}\right), V_{z}^{-1} \varphi \in \mathscr{D}(T)$ and

$$
[T-z S] V_{z}^{-1} \varphi=V_{z}^{-1}\left[T-z^{2}\right] \varphi .
$$

Proof. Follows from a straight-forward computation.

Let $\operatorname{Im} z<0$, i.e. $V_{z}$ is bounded in $L^{2}$ and let us act with it on (6.4)

$$
V_{z}(T-z S) V_{z}^{-1} V_{z} g=V_{z} S f .
$$

Equation (6.6) implies that $V_{z}[T-z S] V_{z}^{-1}=T-z^{2}$ on all functions in $L^{2}$ for which both sides make sense, i.e.

$$
\left(T-z^{2}\right) V_{z} g=V_{z} S f \text {. }
$$

We can now easily solve for $g$, i.e.

$$
R(z) f=V_{z}^{-1}\left(T-z^{2}\right)^{-1} V_{z} S f, \quad \operatorname{Im} z<0 .
$$

For $f \in C_{0}^{\infty}\left(\mathbb{R}^{n}\right)$ we get

$$
\begin{aligned}
(f, R(z) f)_{\mathscr{H}} & =\frac{1}{2}\left(T f, V_{z}^{-1}\left(T-z^{2}\right)^{-1} V_{z} S f\right)_{2}=\frac{1}{2}\left(V_{\bar{z}} T f,\left(T-z^{2}\right)^{-1} V_{z} S f\right)_{2} \\
& =\frac{1}{2}\left(\widehat{V_{\bar{z}} T f},\left(M-z^{2}\right)^{-1} \widehat{V_{z} S f}\right)_{2}, \quad \operatorname{Im} z<0,
\end{aligned}
$$

where denotes the ordinary Fourier transform and $M$ is the multiplication operator $k^{2}+1$. The r.h.s. is analytic in $z$ except for $z^{2} \geqq 1$ and can be analytically continued to all $z \in \mathbb{C} \backslash\left\{z^{2} \geqq 1\right\}$. 
Inserting (6.10) into (6.2) gives

$$
\begin{aligned}
& (f,(E(\beta)-E(\alpha)) f)_{\mathscr{H}}=\int_{\alpha<\omega_{k}<\beta} d k\left|\hat{f}_{+}(k)\right|^{2}, \quad \beta>\alpha>1 \\
& =\int_{-\beta<\omega_{k}<-\alpha} d k\left|\hat{f}_{-}(k)\right|^{2}, \alpha<\beta<-1 \\
& =\int_{\omega_{k}<-\alpha} d k\left|\hat{f}_{-}(k)\right|^{2}+\int_{\omega_{k}<\beta} d k\left|\hat{f}_{+}(k)\right|^{2}, \quad \alpha<-1, \beta>1,
\end{aligned}
$$

where we have defined

$$
\hat{f}_{ \pm}(k)=\frac{1}{2 \omega_{k}} \widehat{V_{ \pm \omega_{k}} T f}(k),
$$

and we have furthermore used that

$$
\frac{1}{2} \widehat{V_{ \pm \omega_{k}} S f_{ \pm}}(k)= \pm \hat{f}_{ \pm}(k) .
$$

In the limit $\alpha \rightarrow-\infty$ and $\beta \rightarrow+\infty$ we get

$$
\|f\|_{\mathscr{H}}^{2}=\left\|\hat{f}_{+}\right\|_{2}^{2}+\left\|\hat{f}_{-}\right\|_{2}^{2}, \quad f \in C_{0}^{\infty}\left(\mathbb{R}^{n}\right) .
$$

Let us define

$$
u_{ \pm}(x, k)=(2 \pi)^{-n / 2} e^{ \pm i \omega_{k}|x|} e^{i k \cdot x},
$$

and let us furthermore for $f, g \in \mathscr{H}$ introduce [compare (4.7)].

$$
2 \sigma(g, f) \equiv(g, S f)_{2}=i \int \frac{d x}{|x|}[(x \cdot \nabla \bar{g}) f-\bar{g}(x \cdot \nabla) f] .
$$

We can then write

$$
\hat{f}_{ \pm}(k)=\frac{1}{\omega_{k}}\left(u_{ \pm}(\cdot, k), f\right)_{\mathscr{H}}= \pm \sigma\left(u_{ \pm}(\cdot, k), f\right)
$$

for $f \in C_{0}^{\infty}\left(\mathbb{R}^{n}\right)$.

Theorem 6.3. The "light-cone Fourier transform" $F: C_{0}^{\infty}\left(\mathbb{R}^{n}\right) \rightarrow L^{2}\left(\mathbb{R}^{n}\right) \oplus L^{2}\left(\mathbb{R}^{n}\right)$ given by $F f=\left\{\hat{f}_{+}, \hat{f}_{-}\right\}$extends to a unitary operator from $\mathscr{H}$ onto $L^{2} \oplus L^{2}$, which diagonalizes $A$

$$
F A f=\left\{\omega_{k} \hat{f}_{+},-\omega_{k} \hat{f}_{-}()\right\}, \quad f \in \mathscr{D}(A) .
$$

Let us then consider the characteristic Cauchy problem

$$
(\square+1) u(x, t)=0, u(x,-|x|)=f(x)
$$

for those $f \in \mathscr{H}$ such that $\hat{f}_{ \pm} \in \mathscr{S}\left(\mathbb{R}^{n}\right)$. The solution then becomes

$$
u(x, t)=(2 \pi)^{-n / 2} \int \frac{d k}{\omega_{k}}\left[e^{-i \omega_{k} t+i k \cdot x} \hat{f}_{+}(k)+e^{i \omega_{k} t+i k \cdot x} \hat{f}_{-}(k)\right] .
$$




\section{The K-G Equation in LC Coordinates and Spectral Theory for $n>1$}

We have so far only considered $n \geqq 3$. In the general case $n \geqq 1$ we are forced to work with sesquilinear forms because of the singular nature of the transformation to $L C$ coordinates. The singularities are worse in lower dimensions and that is why we could manage in a $L^{2}$ setting for $n \geqq 3$.

Let $\varphi \in C_{0}^{\infty}\left(\mathbb{R}^{n+1}\right)$. The K-G equation can then be written

$$
\int d x d t \varphi(\square+1) u=\int d x d t\left\{-\bar{\varphi}_{t} u_{t}+\nabla \bar{\varphi} \cdot \nabla u+\bar{\varphi} u\right\}=0 \text {. }
$$

If we now transform to $L C$ coordinates $(x, \tau)$ we get

$$
\begin{aligned}
& \int d x d \tau\left\{-\tilde{\tilde{\varphi}}_{\tau} \tilde{u}_{\tau}+\left(\nabla+e \frac{\partial}{\partial \tau}\right) \overline{\tilde{\varphi}} \cdot\left(\nabla+e \frac{\partial}{\partial \tau}\right) \tilde{u}+\overline{\tilde{\varphi}} u\right\} \\
& =\int d x d \tau\left\{-\overline{\tilde{\varphi}} e \cdot \nabla \tilde{u}_{\tau}+e \cdot \nabla \overline{\tilde{\varphi}} \tilde{u}_{\tau}+\nabla \overline{\tilde{\varphi}} \cdot \nabla \tilde{u}+\overline{\tilde{\varphi}} \tilde{u}\right\}=0 .
\end{aligned}
$$

Let us from now on drop the . It is sufficient for (7.2) to hold for a finite energy solution $u$ that $; u_{\tau} \in \mathscr{H}$, and

$$
i \frac{d}{d \tau} \sigma(\psi, u(\cdot, \tau))=(\psi, u(\cdot, \tau))_{\mathscr{H}}
$$

for all $\psi \in \mathscr{H}$, where

$$
\sigma(g, f)=\frac{i}{2} \int \frac{d x}{|x|}[(x \cdot \nabla \bar{g}) f-\bar{g}(x \cdot \nabla) f]
$$

for $f, g \in \mathscr{H}$. This form of the K-G equation in LC coordinates makes sense for all $n \geqq 1$.

Proposition 7.1. $\sigma: \mathscr{H} \times \mathscr{H} \rightarrow \mathbb{C}$ is a bounded, symmetric, non-gegenerate sesquilinear form.

Proof. The non-trivial part of the proof is the non-degeneracy. This follows from Proposition 5.4 when $n \geqq 2$, and for $n=1$ it can be verified directly.

This means that there exists a bounded self-adjoint operator $B$ in $\mathscr{H}$ such that

$$
\sigma(g, f)=(g, B f)_{\mathscr{H}},
$$

and the range of $B, \mathscr{R}(B)$ is dense in $\mathscr{H}$.

Definition 7.2. Let $A=B^{-1}$ with $\mathscr{D}(A)=\mathscr{R}(B)$.

$A: \mathscr{D}(A) \rightarrow \mathscr{H}$ is self-adjoint and the solutions of (7.3) in $\mathscr{H}$ are given by $u(\cdot, \tau)$ $=e^{-i A \tau} f, f \in \mathscr{D}(A)$.

Let us then consider the equation $(A-z) g=f, f \in \mathscr{H}, g \in \mathscr{D}(A)$ and $\operatorname{Im} z \neq 0$. This equation is equivalent to

$$
\sigma(h, A g)-z \sigma(h, g)=\sigma(h, f), \quad \forall h \in \mathscr{H},
$$


and because $V_{\bar{z}}^{-1} C_{0}^{\infty}\left(\mathbb{R}^{n}\right)$ is dense in $\mathscr{H}$ we can also write

$$
\left(V_{\bar{z}}^{-1} \varphi, g\right)_{\mathscr{H}}-z \sigma\left(V_{\bar{z}}^{-1} \varphi, g\right)=\sigma\left(V_{\bar{z}}^{-1} \varphi, f\right), \varphi \in C_{0}^{\infty}\left(\mathbb{R}^{n}\right),
$$

where $V_{z}$ be given by (6.5) and we have used (7.5). In complete analogy with (6.6) we have

$$
\left(V_{\bar{z}}^{-1} \varphi, g\right)_{\mathscr{H}}-z \sigma\left(V_{\bar{z}}^{-1} \varphi, g\right)=\left(\varphi, V_{z} g\right)_{\mathscr{H}}-z^{2}\left(\varphi, V_{z} g\right)_{2},
$$

for $\operatorname{Im} z<0$, and (7.7) becomes

$$
\left(\varphi, V_{z} g\right)_{\mathscr{H}}-z^{2}\left(\varphi, V_{z} g\right)_{2}=\sigma\left(V_{\bar{z}}^{-1} \varphi, f\right), \quad \varphi \in C_{0}^{\infty} .
$$

This can be written as

$$
\left(\left(T-\bar{z}^{2}\right) \varphi, V_{z} g\right)_{2}=\sigma\left(V_{\bar{z}}^{-1} \varphi, f\right),
$$

and we get

$$
(\psi, g)_{2}=\sigma\left(V_{\bar{z}}^{-1}\left(T-\bar{z}^{2}\right)^{-1} V_{\bar{z}} \psi, f\right),
$$

where $\psi=V_{z}^{-1}\left(T-z^{2}\right) \varphi$. Let $\xi=T^{-1} \psi$ and then we finally get

$$
(\xi, R(z) f)_{\mathscr{H}}=\sigma\left(V_{\bar{z}}^{-1}\left(T-\bar{z}^{2}\right)^{-1} V_{\bar{z}} T \xi, f\right),
$$

which replaces $(6.10)$ in the general case $n \geqq 1$. The rest then follows in complete analogy with the case $n \geqq 3$.

\section{Conclusions}

We have shown that the characteristic Cauchy problem for the Klein-Gordon equation, $\left(\frac{\partial^{2}}{\partial t^{2}}-\Delta+1\right) u(x, t)=0, u(x,-|x|)=f(x), x \in \mathbb{R}^{n}, n \geqq 1, t \in \mathbb{R}$ has a unique finite energy weak solution for all $f$ such that $\int d x\left(|\nabla f|^{2}+|f|^{2}\right)<\infty$, and furthermore all finite energy weak solutions of the K-G equation are obtained this way. In fact the following equality holds

$$
\begin{aligned}
E & =\frac{1}{2} \int d x\left(\left|u_{t}(x, t)\right|^{2}+|\nabla u(x, t)|^{2}+|u(x, t)|^{2}\right) \\
& =\frac{1}{2} \int d x\left(|\nabla f|^{2}+|f|^{2}\right) .
\end{aligned}
$$

We have developed spectral theory for the K-G equation in light-cone coordinates, including a "light-cone Fourier transform".

There is a Lorentz-invariant symplectic form $\sigma$ on $\mathscr{H}$

$$
\sigma(f, g)=\frac{i}{2} \int \frac{d x}{|x|}\{(x \cdot \nabla \bar{f}) g-\bar{f} x \cdot \nabla g\}=\int \frac{d k}{\omega_{k}}\left\{\overline{\hat{f}}_{+} \hat{g}_{+}-\overline{\hat{f}}_{-} \hat{g}_{-}\right\},
$$

which also is invariant under the unitary light-cone translation group $U(a), a \in \mathbb{R}^{n+1}$ given by

$$
\widehat{U(a) f_{ \pm}}(k)=e^{ \pm i \omega_{k} a_{0}+i k \cdot a} \hat{f}_{ \pm}(k) \text {. }
$$
suggesting improvements and G. Elliott M. Flensted-Jensen and G. Grubb are acknowledged for fruitful discussions. 


\section{References}

1. Hörmander,L.: Linear partial differential operators, p. 151. Berlin, Göttingen, Heidelberg, New York: Springer 1964

2. Riesz,M.: L'intègrale de Riemann-Lionville et le problem de cauchy. Acta Math. 81, 1 (1949)

3. Strichartz, R. S.: Harmonic analysis on hyperboloids. J. Funct. Anal. 12, 341 (1973)

Communicated by H. Araki

Received May 6, 1978 